\title{
Discrete Double Integrator Consensus
}

\author{
David W. Casbeer, Randy Beard, and A. Lee Swindlehurst
}

\begin{abstract}
A distributed double integrator discrete time consensus protocol is presented along with stability analysis. The protocol will achieve consensus when the communication topology contains at least a directed spanning tree. Average consensus is achieved when the communication topology is strongly connected and balanced, where average consensus for double integrator systems is discussed. For second order systems average consensus occurs when the information states tend toward the average of the current information states not their initial values. Lastly, perturbation to the consensus protocol is addressed. Using a designed perturbation input, an algorithm is presented that accurately tracks the center of a vehicle formation in a decentralized manner.
\end{abstract}

\section{INTRODUCTION}

Distributed systems consist of autonomous agents attempting to perform tasks. In order to accomplish any task in an efficient manner there must be some commonly known information. This information is called information states. In most cases, these states are unknown and must be learned, and consensus is one method for each node to learn the information state. In consensus algorithms the agents come into agreement concerning the information state which can take on any value so long as the agents are in accord on that value.

Much work has taken place for first order dynamic consensus [1]-[4], including for discrete time consensus updates [4]-[6]. Discrete time stability analysis is important when communication events take place asynchronously. If the agents communicate fast enough consensus can be approximated by continuous time models. For second order information states, the stability of continuous time consensus has recently been addressed [7]-[10].

Consensus theory has been applied in the control literature to formation control and stability: see [11]-[13] for first order examples and [7], [14]-[16] for second order examples. In general the formation is designed around some reference point and consensus is used to hold the agents in a formation about this reference point. Here rather than specifically controlling the vehicle formations we are simply trying to have each agent come into consensus about some information state. For example, in Section V, we propose an algorithm that accurately tracks the center of the agents' configuration.

This research was supported by the National Science Foundation under Information Technology Research Grant CCF-0428004.

D. Casbeer is a $\mathrm{PhD}$ student with the Dept. of Electrical and Computer Engineering, Brigham Young University, Provo, UT; casbeer abyu . edu

R. Beard is with the faculty of the Dept. of Electrical and Computer Engineering, Brigham Young University, Provo, UT; beard@byu . edu

A.L. Swindlehurst is with the faculty of the Dept. of Electrical Engineering and Computer Science, University of California, Irvine; swindle@uci.edu
In this paper we will present a distributed discrete time double integrator consensus protocol. This work is similar to [7], [10], [14] except here we use discrete time models and extensively use matrix spectral analysis. The paper is organized as follows. In Section II we present the discrete time second order distributed consensus protocol. The spectrum of the transition matrix is presented in Section III. The stability of the consensus protocol is discussed from a geometric viewpoint in Section IV using the spectral analysis of Section III. External inputs are considered in Section IVE. Some examples are presented in Section V; where an algorithm is shown that accurately tracks the center of a vehicle formation. In Section VI some conclusions will be drawn and further research questions are presented.

\section{Second ORder Discrete Time Consensus}

We consider a network of $n$ distinct agents. At any discrete time instant $k$ the interaction topology of the network can be described by the directed graph $\mathcal{G}_{n}[k] \triangleq\left(\mathcal{N}_{n}, \mathcal{E}_{n}[k]\right)$ where $\mathcal{N}_{n}=\{1, \ldots, n\}$ and $\mathcal{E}_{n} \subseteq \mathcal{N}_{n} \times \mathcal{N}_{n}$ are respectively the node set and edge set. The adjacency matrix associated with $\mathcal{G}_{n}[k]$ is given by $\mathbf{A}_{n}[k] \in \mathbb{R}^{n \times n}$, and the $i j^{\text {th }}$ element of $A_{i j}[k]=1$ if there is an edge in the graph connecting $i$ to $j$ otherwise $A_{i j}[k]=0$. From the adjacency matrix we can construct the nonsymmetrical Laplacian matrix $\mathbf{L}_{n}[k] \in$ $\mathbb{R}^{n \times n}$ with $i j^{t h}$ element given by

$$
L_{i j}[k]= \begin{cases}-A_{i j}[k] & \text { if } i \neq j, \\ \sum_{j=1, j \neq i}^{n} A_{i j}[k] & \text { if } i=j .\end{cases}
$$

The Laplacian matrix has at least one zero eigenvalue with an associated eigenvector $\mathbf{1}_{n}$, and all nonzero eigenvalues are in the open right half plane. Also, $\mathbf{L}_{n}[k]$ has exactly one zero eigenvalue if and only if the directed graph $\mathcal{G}_{n}[k]$ has a directed spanning tree [4]. Furthermore, if the graph $\mathcal{G}_{n}[k]$ is balanced, then $\omega_{\ell}=\mathbf{1}_{n}$ is the left eigenvector of the Laplacian matrix that associates with the zero eigenvalue, i.e., $\mathbf{1}_{n}^{T} \mathbf{L}_{n}[k]=0$ [2].

Each agent in the network has an estimate of the double integrator information state $\xi_{i}[k+1]=\xi_{i}[k]+\tau_{k} \zeta_{i}[k], \zeta_{i}[k+$ $1]=\zeta_{i}[k]+\tau_{k} u_{i}[k]$ for $i=1, \ldots, n$. Since, $\tau_{k}>0$ is analogous to a sampling interval, in the following we will refer to it as such. We will use the distributed consensus algorithm (cf. [10])

$$
u_{i}[k]=-\sum_{j=1}^{n} A_{i j}[k]\left[\left(\xi_{i}-\xi_{j}\right)+\gamma_{t}\left(\zeta_{i}-\zeta_{j}\right)\right]
$$


where we drop the time index $k$ when it is clear from the context. The resulting discrete time update becomes

$$
\left[\begin{array}{l}
\boldsymbol{\xi}[k] \\
\boldsymbol{\zeta}[k]
\end{array}\right]=\Psi[k] \otimes \mathbf{I}_{m}\left[\begin{array}{l}
\boldsymbol{\xi}[k-1] \\
\boldsymbol{\zeta}[k-1]
\end{array}\right]
$$

where,

$$
\begin{aligned}
& \Psi[k] \triangleq \mathbf{I}_{2 n}+\tau_{k} \Theta[k], \\
& \Theta[k] \triangleq\left[\begin{array}{cc}
0_{n \times n} & \mathbf{I}_{n} \\
-\mathbf{L}_{n}[k] & -\gamma_{k} \mathbf{L}_{n}[k]
\end{array}\right],
\end{aligned}
$$

and $\gamma_{k}>0$. Each local update in the discrete protocol (3) is

$$
\begin{aligned}
\xi_{i}[k+1]= & \xi_{i}[k]+\tau_{k+1} \zeta_{i}[k] \\
\zeta_{i}[k+1]= & \zeta_{i}[k]-\tau_{k+1} \sum_{j=1}^{n} A_{i j}[k+1] \\
& \cdot\left(\left(\xi_{i}[k]-\xi_{j}[k]\right)+\gamma_{k}\left(\zeta_{i}[k]-\zeta_{j}[k]\right)\right)
\end{aligned}
$$

which shows that (3) is a distributed protocol since local updates are only a function of neighboring agents. In this paper we endeavor to prove that consensus is achieved using the input (2).

\section{Spectrum of $\Psi[k]$}

We will now solve for the eigenvalues and the right and left (generalized) eigenvectors [17] of $\Psi$ in terms of the eigenvalues and eigenvectors of $-\mathbf{L}_{n}$. Before proceeding we define three different sets of eigenvalues and eigenvectors. First, the matrix $-\mathbf{L}_{n}$ has eigenvalues $\lambda_{i}$ with corresponding right and left eigenvectors given by $\mathbf{w}_{r, i}$ and $\mathbf{w}_{\ell, i}$ for $i=$ $1, \ldots, n$. Examining the relationship between $\Psi$ and $\Theta$ in (4) we notice that

$$
\rho_{i}=\tau_{k} \theta_{i}+1,
$$

where $\rho_{i}$ and $\theta_{i}$ are the $i^{\text {th }}$ eigenvalue for $\Psi$ and $\Theta$ respectively, and the eigenvectors for $\Psi$ are the same as those of $\Theta$. From this fact, denote the $i^{\text {th }}$ right and left (generalized) eigenvectors of these matrices respectively by $\mathbf{m}_{i}$ and $\mathbf{v}_{i}$ for $i=1, \ldots, 2 n$.

Normally, the left eigenvectors and left generalized eigenvectors of a matrix are found by taking the inverse of a matrix $\mathbf{M}$ which has columns consisting of the right (generalized) eigenvectors. The rows of the result $\mathbf{V}=\mathbf{M}^{-1}$ are then the left (generalized) eigenvectors. In this paper, we need to know at least the left eigenvector and left generalized eigenvector associated with the eigenvalue $\theta=0$. We will only present the derivation of the left (generalized) eigenvectors.

It is assumed that the graph $\mathcal{G}_{n}$ contains a directed spanning tree which according to the properties of the Laplacian matrix given at the beginning of Sec. II, ensures that $\lambda_{1}=0$ is a simple eigenvalue of $-\mathbf{L}_{n}$ with corresponding right eigenvector $\mathbf{w}_{r, 1}=\mathbf{1}_{n}$. We proceed by finding the right eigenvectors and right generalized eigenvectors of $\Theta$. Then we find the eigenvalues and left eigenvectors and left generalized eigenvectors of $\Theta$.

\section{A. Right Eigenvectors}

The relationship between the eigenvalues of $\Theta$ and the Laplacian matrix $\mathbf{L}$ is $\lambda_{j}=\theta_{i}^{2} /\left(1+\theta_{i} \gamma\right)$ for $i=2 j-1,2 j$ and $j=1, \ldots, n$ (cf. (13)). The right eigenvectors of $\Theta$ corresponding to eigenvalues $\theta_{i} \neq 0$ are given by

$$
\mathbf{m}_{i}=\left[\begin{array}{ll}
\mathbf{w}_{r, j}^{T} & \theta_{i} \mathbf{w}_{r, j}^{T}
\end{array}\right]^{T}
$$

for $i=2 j-1,2 j$ and $j=2, \ldots, n .^{1}$ The right eigenvector and generalized right eigenvector corresponding to the eigenvalues $\theta_{1}=\theta_{2}=0$ are given respectively by

$$
\mathbf{m}_{1}=\left[\begin{array}{l}
\mathbf{1}_{n} \\
\mathbf{0}_{n}
\end{array}\right] \quad \text { and } \quad \mathbf{m}_{2}=\left[\begin{array}{l}
\mathbf{0}_{n} \\
\mathbf{1}_{n}
\end{array}\right]
$$

If there is a repeated eigenvalue $\lambda_{j}=\lambda_{i} \neq 0$, then the generalized eigenvector needs to be found.

\section{B. Left Eigenvectors and Corresponding Eigenvalues}

In order to solve for the left eigenvectors and corresponding eigenvalues of $\Theta$ sub-divide $\mathbf{v}_{i}$ as $\mathbf{v}_{i}=\left[\begin{array}{ll}v_{i, a}^{T} & v_{i, b}^{T}\end{array}\right]^{T}$. $\mathbf{v}_{i}$ and $\theta_{i}$ can be found by solving the characteristic equation $\left(\Theta^{T}-\theta_{i} \mathbf{I}_{2 n}\right) \mathbf{v}_{i}=\mathbf{0}_{2 n}$. Multiplying out this equation in terms of $v_{i, a}$ and $v_{i, b}$ yields the two matrix equations

$$
\begin{aligned}
-\mathbf{L}_{n}^{T} v_{i, b} & =\theta_{i} v_{i, a} \\
v_{i, a}-\gamma \mathbf{L}_{n}^{T} v_{i, b} & =\theta_{i} v_{i, b} .
\end{aligned} \quad \text { and, }
$$

Manipulating (11) and (12) for one equation in terms of only $v_{i, b}$ yields

$$
-\mathbf{L}_{n}^{T} v_{i, b}=\frac{\theta_{i}^{2}}{\left(1+\theta_{i} \gamma\right)} v_{i, b},
$$

which is simply the left eigenvector equation for the negative of the Laplacian with $\lambda_{j}=\theta_{i}^{2} /\left(1+\theta_{i} \gamma\right)$.

Thus for any $\lambda_{j}$ with corresponding left eigenvector $\mathbf{w}_{\ell, j}$, $\Theta$ has two eigenvalues, $\theta_{2 j-1}$ and $\theta_{2 j}$, that are the two solutions to the equation

$$
\theta_{ \pm}=\frac{1}{2}\left(\gamma \lambda_{j} \pm \sqrt{\gamma^{2} \lambda_{j}^{2}+4 \lambda_{j}}\right)
$$

and $v_{i, b}=\sigma_{i} \mathbf{w}_{\ell, j}$ for $i=2 j-1,2 j$ with any scale factor $\sigma_{i}$. Plugging the resulting $\theta_{i, b}$ and $v_{i, b}$ back into (11) gives

$$
\sigma_{i} \lambda_{j} \mathbf{w}_{\ell, j}=\theta_{i} v_{i, a} \text { for } i=2 j-1,2 j .
$$

When $\lambda_{j} \neq 0$

$$
\mathbf{v}_{i}=\sigma_{i}\left[\begin{array}{c}
\frac{\lambda_{j}}{\theta_{i}} \mathbf{w}_{\ell, j} \\
\mathbf{w}_{\ell, j}
\end{array}\right] \quad \text { for } i=2 j-1,2 j .
$$

However when $\lambda_{j}=0$ this will not work. In which case we use (12) which gives $v_{i, a}=\mathbf{0}_{n}$ for $i=1,2$.

The geometric multiplicity of the zero eigenvalue is one. Also, the algebraic multiplicity of the zero eigenvalue for $\Theta$ is two (i.e., $\theta_{1}=\theta_{2}=0$ ). This is because there are two solutions to (14) for the simple eigenvalue $\lambda_{1}=0$ because of the assumption that the graph $\mathcal{G}_{n}$ have a directed spanning

\footnotetext{
${ }^{1}$ This assumes there are no repeated eigenvalues $\lambda_{i}$ for $-\mathbf{L}_{n}$. If there are repeated eigenvalues with geometric multiplicity less than the algebraic multiplicity then generalized eigenvectors must be found. See the derivation of the left generalized eigenvectors in III-B for how this is done.
} 
tree. Such a scenario requires the second eigenvector associated with the zero eigenvalue to be a generalized eigenvector [17].

Let $\theta_{1}=\theta_{2}=0$ and $\mathbf{v}_{2}=\sigma_{2}\left[\begin{array}{ll}\mathbf{0}_{n}^{T} & \mathbf{w}_{\ell, 1}^{T}\end{array}\right]^{T}$ where $\mathbf{w}_{\ell, 1}$ is associated with the eigenvalue $\lambda_{1}=0$. The generalized eigenvector $\mathbf{v}_{1}$ is found by solving

$$
\left(\Theta^{T}-\theta_{2} \mathbf{I}_{2 n}\right) \mathbf{v}_{1}=\mathbf{v}_{2},
$$

which after some algebra becomes $\mathbf{v}_{1}=\sigma_{1}\left[\begin{array}{ll}\mathbf{w}_{\ell, 1}^{T} & \alpha \mathbf{w}_{\ell, 1}^{T}\end{array}\right]^{T}$ where the unknown scalar $\alpha$ is chosen to be $\alpha=0$. This choice for $\alpha$ is motivated from two rationale: it is simple, and it maintains orthogonality relations with the right (generalized) eigenvectors which turns out to be helpful subsequently.

The indexing for $\mathbf{v}_{1}$ and $\mathbf{v}_{2}$ may seem obtuse; there is a reasonable explanation. The left eigenvectors are normally found by taking the inverse of the matrix whose columns are the right eigenvectors, which causes $\mathbf{v}_{j}^{T} \mathbf{m}_{i}=0$ for $i \neq j$ and $\mathbf{v}_{j}^{T} \mathbf{m}_{j}=1$. The indexing for $\mathbf{v}_{1}$ and $\mathbf{v}_{2}$ was chosen to maintain this relationship. Lastly, so that orthonormality is preserved, we set

$$
\sigma_{i}=\left(\mathbf{v}_{i}^{T} \mathbf{m}_{i}\right)^{-1} .
$$

If by chance, there is another repeated eigenvalue $\lambda_{j} \neq 0$ then the same process can be repeated to find the appropriate generalized left eigenvectors. We refrain from this exercise since it is not pertinent to the results of this paper.

\section{Stability Analysis}

Using the eigenvalues and (generalized) eigenvectors found in the previous section, we write a similarity transformation for the matrix $\Psi=\mathrm{MJV}$ where:

$$
\begin{aligned}
& \mathbf{M} \triangleq\left[\begin{array}{lll}
\mathbf{m}_{1} & \cdots & \mathbf{m}_{2 n}
\end{array}\right], \\
& \mathbf{V} \triangleq \mathbf{M}^{-1}=\left[\begin{array}{lll}
\mathbf{v}_{1} & \cdots & \mathbf{v}_{2 n}
\end{array}\right]^{T},
\end{aligned}
$$

and $\mathbf{J}$ is in Jordan normal form with diagonal elements $\rho_{i}$ for $i=1, \ldots, 2 n$. To facilitate our investigation of average consensus, the matrices $\mathbf{M}, \mathbf{V}$, and $\mathbf{J}$ are partitioned into two invariant sets. One partition is associated with the eigenvalues $\rho_{1}=\rho_{2}=1$; these eigenvalues correspond to the Laplacian eigenvector $\mathbf{w}_{r, 1}$. The other partition is associated with all other eigenvalues. These partitions are written as $\mathbf{M}=\left[M_{1} \vdots M_{2}\right], \mathbf{V}=\left[\begin{array}{ll}V_{1}^{T} & V_{2}^{T}\end{array}\right]^{T}$, and

$$
\mathbf{J}=\left[\begin{array}{c:c}
J_{1} & \mathbf{0}_{2 \times 2} \\
\hdashline \mathbf{0}_{2 n-2 \times 2 n-2} & J_{2}
\end{array}\right]
$$

where $M_{1}=\left[\mathbf{m}_{1} \mathbf{m}_{2}\right], M_{2}=\left[\mathbf{m}_{3} \cdots \mathbf{m}_{2 n}\right], V_{1}=\left[\mathbf{v}_{1}^{T} \mathbf{v}_{2}^{T}\right]^{T}$, $V_{2}=\left[\mathbf{v}_{3}^{T} \cdots \mathbf{v}_{2 n}^{T}\right]^{T}$,

$$
J_{1}=\left[\begin{array}{ll}
1 & \tau \\
0 & 1
\end{array}\right]
$$

and $J_{2}$ is the Jordan matrix comprising all the Jordan blocks from eigenvalues $\rho_{i}$ for $i=3, \ldots, n$. With these definitions we can rewrite the matrix $\Psi=M_{1} J_{1} V_{1}+M_{2} J_{2} V_{2}$.
Let $\Phi[k, k-j]$ be the transition matrix,

$$
\Phi[k, k-j] \triangleq(\Psi[k] \Psi[k-1] \cdots \Psi[k-j+1]) \otimes \mathbf{I}_{m} .
$$

The transition matrix propagates the information states from time $k-j$ to $k$. By looking at the transition matrix we will be able to determine if consensus is achieved. Also, define the set $\mathcal{P}[k]=\left\{\rho_{i}[k] \mid \rho_{i}[k] \neq 1, i=1, \ldots, 2 n\right\}$, e.g., $\mathcal{P}[k]$ contains all eigenvalues of $\Psi[k]$ that are not one, where the eigenvalues $\rho_{i}[k]$ of $\Psi[k]$ were defined in (8). This set contains the eigenvalues in the partition $M_{2} J_{2} V_{2}$.

\section{A. Constant Communication Topology}

Let $\Psi[k]$ be constant $e . g$., $\Psi[k]=\Psi$, with $\tau=t_{k}-t_{k-1}$ for all $k \geq 1$. The transition matrix becomes

$$
\begin{aligned}
\Phi[k, 0] & =\mathbf{M} \mathbf{J}^{k} \mathbf{V} \otimes \mathbf{I}_{m} \\
& =\left(M_{1} J_{1}^{k} V_{1}+M_{2} J_{2}^{k} V_{2}\right) \otimes \mathbf{I}_{m} .
\end{aligned}
$$

To see this fact, note that $\mathbf{V}=\mathbf{M}^{-1}$. Thus $(\mathbf{M J V})^{2}=$ $\mathbf{M J}^{2} \mathbf{V}$.

By definition, the set $\mathcal{P}$ contains the eigenvalues of $M_{2} J_{2} V_{2}$. Because, all the elements of $\mathcal{P}$ have a modulus less than one the matrix $M_{2} J_{2} V_{2}$ is (discrete-time) stable. ${ }^{2}$ From this fact it can be seen that $\Phi[k, 0] \rightarrow M_{1} J_{1}^{k} V_{1} \otimes \mathbf{I}_{m}$ as $k \rightarrow \infty$, which allows us to show that consensus is achieved in the following Lemma.

Lemma 1. (cf. [10]) Assuming the graph $\mathcal{G}_{n}$ has a directed spanning tree and all elements of the set $\mathcal{P}$ have a modulus less than one, then the consensus protocol given in (3) will achieve consensus asymptotically.

Proof. With the given assumptions, $\mathbf{x}_{k+1}=M_{2} J_{2} V_{2} \mathbf{x}_{k}$ is asymptotically stable which says $\Phi[k, 0] \rightarrow M_{1} J_{1}^{k} V_{1} \otimes \mathbf{I}_{m}$ asymptotically as $k \rightarrow \infty$. Since,

$$
M_{1} J_{1}^{k} V_{1}=\left[\begin{array}{cc}
\mathbf{1}_{n} \mathbf{w}_{\ell, 1}^{T} & k \tau \mathbf{1}_{n} \mathbf{w}_{\ell, 1}^{T} \\
\mathbf{0}_{n, n} & \mathbf{1}_{n} \mathbf{w}_{\ell, 1}^{T}
\end{array}\right]
$$

we can see that $\xi[k] \rightarrow\left(\mathbf{1}_{n} \mathbf{w}_{\ell, 1}^{T} \otimes \mathbf{I}_{m}\right) \xi[0]+k \tau\left(\mathbf{1}_{n} \mathbf{w}_{\ell, 1}^{T} \otimes\right.$ $\left.\mathbf{I}_{m}\right) \boldsymbol{\zeta}[0]$ and $\boldsymbol{\zeta}[k] \rightarrow\left(\mathbf{1}_{n} \mathbf{w}_{\ell, 1}^{T} \otimes \mathbf{I}_{m}\right) \boldsymbol{\zeta}[0]$ as $k$ gets large. Consequently, $\left\|\xi_{i}[k]-\xi_{j}[k]\right\| \rightarrow 0$ and $\left\|\zeta_{i}[k]-\zeta_{j}[k]\right\| \rightarrow 0$ for all $i, j$ and $k \rightarrow \infty$, meaning consensus is achieved at the rate of decay of $M_{2} J_{2}^{k} V_{2}$.

Notice, that the final consensus value is a weighted average, with the weighting coming from the eigenvector $\mathbf{w}_{\ell, 1}$ of the Laplacian $-\mathbf{L}_{n}$.

\section{B. Switching Communication Topologies}

In the case of switching communication topologies we will need to state a few Lemmas in order to prove consensus.

Lemma 2. Let the one step transition matrices associated with the directed graphs $\mathcal{G}_{n}[k]$ and $\mathcal{G}_{n}[j]$ be respectively $\Psi[k]$ and $\Psi[j]$. When both graphs $\mathcal{G}_{n}[k]$ and $\mathcal{G}_{n}[j]$ are strongly

\footnotetext{
${ }^{2}$ A continuously evolving matrix is stable if the real part of all its eigenvalues is negative. A matrix is stable in discrete time if the modulus of all its eigenvalues is less than one.
} 
connected and balanced the matrix partitions of $\Psi[k]$ and $\Psi[j]$ have the following properties

$$
\begin{aligned}
& V_{1}[k] M_{1}[j]=\mathbf{I}_{2} \\
& V_{1}[k] M_{2}[j]=\mathbf{0}_{2 \times(2 n-2)} \\
& V_{2}[k] M_{1}[j]=\mathbf{0}_{(2 n-2) \times 2} .
\end{aligned}
$$

Corollary 1. When both directed graphs $\mathcal{G}_{n}[k]$ and $\mathcal{G}_{n}[j]$ contain a directed spanning tree but are not strongly connected and balanced, the matrix partitions of $\Psi[k]$ and $\Psi[j]$ will have properties (27) and (28) but not (29).

Let $\Theta[k]$ come from the bounded and closed set $\left\{\Theta_{\sigma} \mid \sigma \in\right.$ $\mathcal{S}\}$ where $\mathcal{S}$ is the index set for all possible matrices $\Theta_{\sigma}$. Given some $\sigma$ let

$$
\theta_{\sigma, \min } \triangleq \underset{\theta_{i, \sigma} \neq 0}{\arg \min }\left\{-\frac{2 \operatorname{Re}\left(\theta_{i, \sigma}\right)}{\left|\theta_{i, \sigma}\right|^{2}}\right\}
$$

where $\theta_{i, \sigma}$ are the eigenvalues of $\Theta_{\sigma}$. Let $s_{k} \in \mathcal{S}$ denote the switching signal for the sampling interval $\tau_{k}$; consequently, we can think of $s_{k}$ as choosing the active graph configuration and matrix $\Psi[k]$. The number of sequential time intervals over which $s_{k}$ does not change is called the dwell time and is denoted by $\kappa$ [18]. With these definitions we write the following Lemma.

Lemma 3. ${ }^{3}$ Fix the number of agents $n$. Let the scalar gain $\gamma_{\sigma}$ in $\Theta_{\sigma}$ be bounded by two finite positive constants $\gamma_{L B}$ and $\gamma_{U B}$ such that

$$
0<\gamma_{L B} \leq \gamma_{k} \leq \gamma_{U B}<\infty .
$$

Also, suppose the sampling interval $\tau_{\sigma}$ (used to define $\Psi_{\sigma}$ from $\left.\Theta_{\sigma}\right)$ is bounded by finite positive constants $\tau_{L B}$ and $\tau_{U B}$ such that

$$
0<\tau_{L B} \leq \tau_{\sigma} \leq \tau_{U B}<-\frac{2 \operatorname{Re}\left(\theta_{\sigma, \min }\right)}{\left|\theta_{\sigma, \min }\right|^{2}} .
$$

Under these assumptions the set of all possible matrix partitions $\left\{M_{2, \sigma} J_{2, \sigma} V_{2, \sigma} \mid \sigma \in \mathcal{S}\right\}$ is closed and bounded; furthermore, all matrices in this set are stable. Let $a_{\sigma}$ and $\chi_{\sigma}$ be any finite, nonnegative and positive number, respectively, for which

$$
\left\|M_{2, \sigma} J_{2, \sigma}^{k} V_{2, \sigma}\right\| \leq e^{a_{\sigma}-\chi_{\sigma} k}, k=0,1,2, \ldots .
$$

Suppose also that some nonnegative integer $\kappa_{0}$ exists such that $\kappa_{0}>\sup _{\sigma \in \mathcal{S}}\left\{\frac{a_{\sigma}}{\chi_{\sigma}}\right\}$. Then for any admissible switching

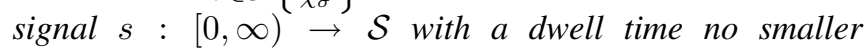
than $\kappa_{0}$, the transition matrix of $M_{2, \sigma} J_{2, \sigma} V_{2, \sigma}$ satisfies $\left\|\Phi_{2}\left[k, k_{0}\right]\right\| \leq e^{a-\chi\left(k-k_{0}\right)}, \forall k \geq k_{0} \geq 0$, where $a=$ $\sup _{\sigma \in \mathcal{S}}\left\{a_{\sigma}\right\}, \chi=\inf _{\sigma \in \mathcal{S}}\left\{\chi_{\sigma}-\frac{a_{\sigma}}{\kappa_{0}}\right\}$, and $\Phi_{2}\left[k, k_{0}\right]=$ $M_{2}[k] J_{2}[k] V_{2}[k] \cdots M_{2}\left[k_{0}\right] J_{2}\left[k_{0}\right] V_{2}\left[k_{0}\right]$.

Proof of Lemma 3. To see that all matrices $M_{2, \sigma} J_{2, \sigma} V_{2, \sigma}$ in $\left\{M_{2, \sigma} J_{2, \sigma} V_{2, \sigma} \mid \sigma \in \mathcal{S}\right\}$ are stable note that if $\tau_{\sigma}$ satisfies (32) then we know $1>\left|\rho_{i, \sigma}\right|=\left|\tau_{\sigma} \theta_{i, \sigma}+1\right|$ which becomes

$$
\tau_{\sigma}<-\frac{2 \operatorname{Re}\left(\theta_{i, \sigma}\right)}{\left|\theta_{i, \sigma}\right|^{2}}, \forall i=3, \ldots, 2 n,
$$

\footnotetext{
${ }^{3}$ This Lemma and its proof are motivated from Lemma 2 in [18]
}

where $\rho_{i, \sigma}$ are the eigenvalues of the matrix $\Psi_{\sigma}$ formed from $\Theta_{\sigma}$. Thus the matrix $M_{2, \sigma} J_{2, \sigma} V_{2, \sigma}$ is stable and (33) holds.

Fixing $n$ along with (31) imply the set $\left\{\Theta_{\sigma} \mid \sigma \in\right.$ $\mathcal{S}\}$ is closed and bounded; this fact combined with the bound in (32) implies that the set $\left\{\Psi_{\sigma} \mid \sigma \in \mathcal{S}\right\}$ is also closed and bounded. Thus the set of all matrix partitions $\left\{M_{2, \sigma} J_{2, \sigma} V_{2, \sigma} \mid \sigma \in \mathcal{S}\right\}$ is closed and bounded.

The rest of the proof parallels the proof of Lemma 2 in [18] using discrete time steps rather than continuous.

Notice that the time between communication events must be bounded from above according to (32); this ensures that the matrix $\Psi[k]$ is stable. This Lemma says the switching signal $s_{k}$ must dwell long enough on the values of $\mathcal{S}$ so that the transition matrix $\Phi_{2}$ can drop far enough in value (i.e., at least $\kappa_{0}$ time steps). We can now state our first Lemma about switching consensus.

Lemma 4. If the graphs $\mathcal{G}_{n}[k]$ are strongly connected and balanced, $\Theta[k]$ is stable in the continuous time sense (i.e., $\operatorname{Re}\left(\theta_{i}[k]\right) \leq 0$ for all $i=1, \ldots, 2 n$ ). If $\tau_{k}$ satisfies (32) and the dwell time $\kappa$ of every graph $\mathcal{G}_{n}$ satisfies $\kappa>\sup _{\sigma \in \mathcal{S}}\left\{\frac{a_{\sigma}}{\chi_{\sigma}}\right\}$, then protocol (3) achieves consensus asymptotically.

Proof of Lemma 4. Assume that all graphs $\mathcal{G}_{n}[k] k=$ $1, \ldots, \infty$ are strongly connected and balanced. As a result of Lemma 2 the information state transition matrix becomes

$\Phi[k, 0]=\left(M_{1}[k]\left(J_{1}[k] \cdots J_{1}[0]\right) V_{1}[0]+\Phi_{2}[k, 0]\right) \otimes \mathbf{I}_{m}$.

There were three assumptions in the proposition: 1) $\Theta[k]$ is stable, 2) $\tau_{k}$ satisfies (32), and 3) the dwell time of every graph $\mathcal{G}_{n}$ satisfies $\kappa>\sup _{\sigma \in \mathcal{S}}\left\{\frac{a_{\sigma}}{\chi_{\sigma}}\right\}$. Under these assumptions we know the transition matrix $\Phi_{2}[k, 0]$ in (35) is stable because of Lemma 3 .

The term $M_{1}[k]\left(J_{1}[k] \cdots J_{1}[0]\right) V_{1}[0]$ in (35) can be written in the form of (26) by replacing $k \tau$ in the upper right hand quadrant by $\sum_{j=0}^{k} \tau_{j}$. As a consequence of this and the fact that $\Phi_{2}[k, 0]$ defines an asymptotically stable system, the protocol (3) achieves consensus asymptotically.

We can also state a proof in the case of graphs which contain a directed spanning tree.

Lemma 5. If the directed graphs $\mathcal{G}_{n}[k]$ contain a directed spanning tree, $\Theta[k]$ is stable in the continuous time sense (i.e., $\operatorname{Re}\left(\theta_{i}[k]\right) \leq 0$ for all $i=1, \ldots, 2 n$ ), $\tau_{k}$ satisfies (32) for all $k=1, \ldots, \infty$, and $\kappa$ satisfies $\kappa>\sup _{\sigma \in \mathcal{S}}\left\{\frac{a_{\sigma}}{\chi_{\sigma}}\right\}$, then protocol (3) achieves consensus asymptotically.

Proof. Assume that all graphs $\mathcal{G}_{n}[k] k=1, \ldots, \infty$ contain a directed spanning tree. As a result of Corollary 1 the information state transition matrix becomes

$$
\Phi[k, 0]=\left(M_{1} \sum_{k=0} \Gamma_{k}+\Phi_{2}[k, 0]\right) \otimes \mathbf{I}_{m}
$$


where $\Phi_{2}[k, 0]$ is asymptotically stable as was shown in the proof of 4. After some algebra is can be verified that each block quadrant of, $M_{1} \sum_{k=0} \Gamma_{k}$ has the form

$$
M_{1} \sum_{k=0} \Gamma_{k}=\left[\begin{array}{ll}
\mathbf{1}_{n} \mathbf{q}_{a} & \mathbf{1}_{n} \mathbf{q}_{d} \\
\mathbf{1}_{n} \mathbf{q}_{b} & \mathbf{1}_{n} \mathbf{q}_{c}
\end{array}\right] .
$$

Following the logic in the proof of Lemma 1 consensus is achieved asymptotically.

\section{Average Consensus}

Looking at the constant matrix $\Psi$ and switching matrices $\Psi[k]$ that are all strongly connected and balanced graphs (Lemma 1 with strongly connected and balanced graph and Lemma 4), we can see that a type of average consensus is achieved. This is because $\mathbf{w}_{\ell, 1}=\mathbf{1}_{n}$ in (26) when the graphs are strongly connected and balanced graphs.

In this situation the information state derivatives will all tend toward the average of the initial $\zeta_{i}$, for $i=1, \ldots, n, e . g$, $\zeta_{i}[k] \rightarrow \sum_{i=1}^{n} \zeta_{i}[0]$. The information states at each node, however, tend toward the average of the initial states plus a scaling of the average information state derivatives, $\xi_{i}[k] \rightarrow$ $\sum_{i=1}^{n} \xi_{i}[0]+\sum_{j=0}^{k} \tau_{k} \sum_{i=1}^{n} \zeta_{i}[0]$.

This is not exactly average consensus as it is understood for information states with first order dynamics, yet it has desirable properties. The information state derivatives $\zeta_{i}[k]$ tend to the average of their initial values, while the information state $\xi_{i}[k]$ tend to the average of information states at the current time, namely

$$
\xi_{i}[k] \rightarrow \sum_{i=0}^{n} \xi_{i}[k]=\sum_{i=0}^{n}\left(\xi_{i}[0]+\zeta_{i}[k] \sum_{j=0}^{k} \tau_{j}\right) .
$$

Notice also that any value of $\tau$ such that $-2 \operatorname{Re}\left(\theta_{j}\right) /\left|\theta_{j}\right|^{2}>\tau>0$ for all $j=3, \ldots, 2 n$ will reach consensus.

\section{Eigenvalue properties}

All of the consensus proofs rely on the fact that $\left|\rho_{i}\right|<1$ for all $\rho_{i} \in \mathcal{P}$; a necessary condition for $\left|\rho_{i}\right|<1$ is that $\tau$ satisfies (32) which implies $\Theta$ is stable, e.g., $\operatorname{Re}\left(\theta_{i}\right)<0$. To ensure that the eigenvalues of $\Theta$ have negative real parts the variable $\gamma$ must be bounded by

$$
\gamma>\max _{i=2, \ldots, n} \sqrt{\frac{2}{\left|\lambda_{i}\right| \cos \left[\tan ^{-1} \frac{\operatorname{Im}\left(\lambda_{i}\right)}{-\operatorname{Re}\left(\lambda_{i}\right)}\right]}}
$$

(see [10]). Looking back to (32), we can see that if the sampling interval $\tau$ becomes too large then it is possible that no such $\gamma$ exists as can be seen in Figure 1. This plot varies $\gamma$ for some network topology $-\mathbf{L}$. Two different values of $\tau$ are plotted and for the larger of the two $\tau=.8$ there is no $\gamma$ that exists to ensure $\Psi$ is stable.

At present, we have been unable to find a technique for bounding $\gamma$ given a graph $\mathcal{G}_{n}[k]$ and sampling interval $\tau_{k}$ where the graph has at least a directed spanning tree and the sampling interval is such that stability is feasible.

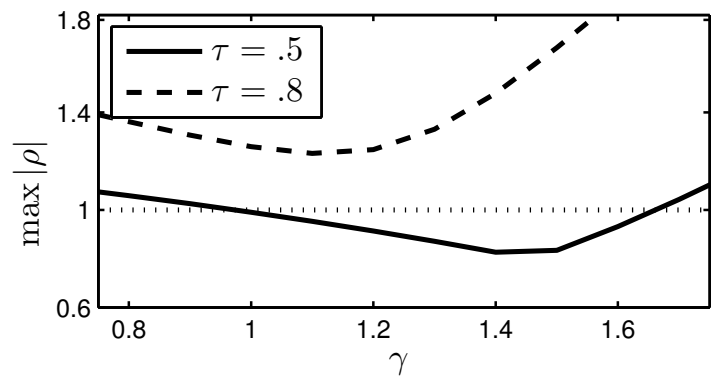

Fig. 1. Here $\gamma$ is varied for two different sampling intervals $\tau=.5$ and .8 seconds. If $\tau$ is too long there is no $\gamma$ ensuring asymptotic consensus since at least one eigenvalue $\rho_{i} \in \mathcal{P}$ has a modulus greater than one.

\section{E. External Stability}

The consensus protocol (3) can be extended to include bounded perturbations $\mathbf{w}[k]$

$$
\left[\begin{array}{l}
\boldsymbol{\xi}[k] \\
\boldsymbol{\zeta}[k]
\end{array}\right]=\Psi[k] \otimes \mathbf{I}_{m}\left[\begin{array}{l}
\boldsymbol{\xi}[k-1] \\
\boldsymbol{\zeta}[k-1]
\end{array}\right]+\mathbf{w}[k] .
$$

In all the previously mentioned scenarios the zero state solution is exponentially stable (since asymptotic and exponential stability are synonymous in discrete time [19]). Because of this, we know that (40) is bounded-input bounded-state stable. Thus given a bounded input the agents will achieve consensus within some bound $\left\|\xi_{i}[k]-\xi_{j}[k]\right\| \leq c$ as $k \rightarrow \infty$ for some finite scalar $c$. In Section V, a known (bounded) input $\mathbf{w}[k]$ is employed to accurately track the center of the agents' configuration.

\section{Simulations}

The five communication topologies used are shown in Fig. 2 with the exception of Graph 5 which does not contain a spanning tree. It can be derived from Graph 4 by disconnecting Nodes 3 and 4.

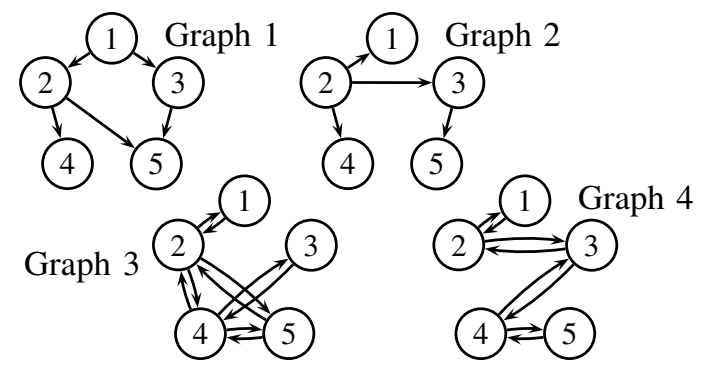

Fig. 2. The four of the five graphs used for the simulations. Graph 5 is formed by removing both links between Nodes 3 and 4 in Graph 4.

The first two examples use a constant sampling interval $\tau=.5$ seconds and run for fifty iterations. They employ scalar second order dynamic systems as in (3). In the first example Graph 5 was used $100 \%$ of the time. The results are shown in Fig. 3. Notice this system really is two separate systems where each system individually achieves consensus.

The second example involves switching between two graphs (Graph $133 \%$ of the time and Graph 2 uses the remaining time). In Fig. 4 it can be verified that the nodes achieve consensus. Notice that the "average" consensus (the black line in the figure) is not achieved because Graphs 1 and 2 are not strongly balanced, $c f$. Section IV-C. 


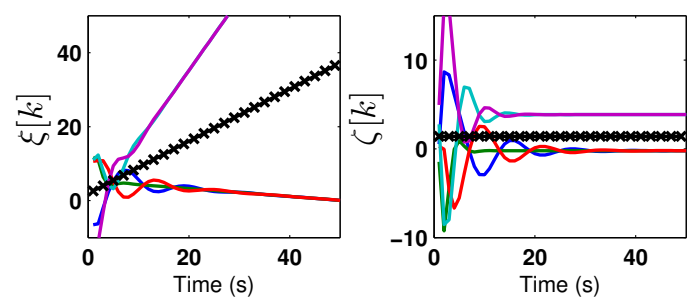

Fig. 3. Unconnected graph. $\xi$ is on the left and $\zeta$ is on the right. The black line with the marks is the true average state (see (38)). The other lines are each nodes' conjecture of the information state or its derivative.
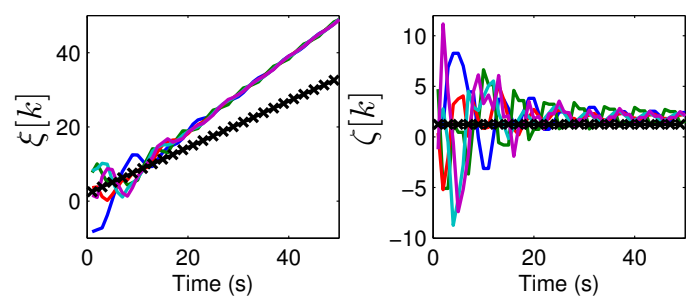

Fig. 4. Switching between Graphs 1 and 2, each containing a directed spanning tree. The black line with the marks is the true average state. The other lines are each nodes' conjecture of the information state or its derivative.

The last simulation proceeds for 100 seconds with $\tau=.5$. Graph 4 is utilized $66 \%$ of the time with Graph 3 using the remaining time. A two dimensional information state $\boldsymbol{\xi}[k]$ is utilized that is the center of the formation. Each node travels at constant velocity of $1 \mathrm{~m} / \mathrm{s}$ and at every time step each nodes' heading randomly changes uniformly from $\left[-\frac{\pi}{4}, \frac{\pi}{4}\right]$. Initially, each node's information state is its own position. Each node also can measure its velocity $\dot{\mathbf{x}}_{i}[k]$; at time $k=0$ the term $\zeta_{i}[0]=\dot{\mathbf{x}}_{i}[0]$. The input is given by the change in heading. Denoting a nodes' velocity at time $k$ by the vector $\mathbf{v}_{i}[k]=\left[v_{x, i}[k] v_{y, i}[k]\right]^{T}$, the perturbation term for the information state is written as $\mathbf{w}[k]=\left[\mathbf{0}_{m n}^{T} \delta \mathbf{v}_{1}^{T}[k] \cdots \delta \mathbf{v}_{n}^{T}[k]\right]^{T}$ where $\delta \mathbf{v}_{i}^{T}[k]=\mathbf{v}_{i}[k]-\mathbf{v}_{i}[k-1]$. Using this algorithm the nodes are able to accurately track the center in a distributed fashion. In Fig. 5 we have plotted the $x$ axis information.
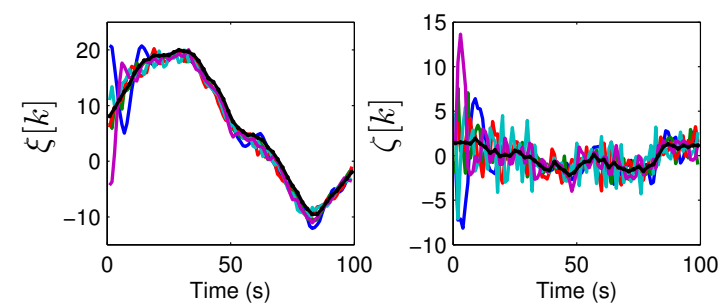

Fig. 5. Tracking the center of the agents using a perturbation term. Here $\xi$ and $\zeta$ are respectively the $x$ position and velocity of the center. The black line is the true average state.

\section{CONCLUSIONS}

In this paper we have presented the discrete time second order distributed consensus protocol (3). Under certain assumptions this protocol has been shown to achieve asymptotic consensus for switching networks that all contain a directed spanning tree; Furthermore, (when the conditions are met) for strongly connected and balanced networks, (3) achieves average consensus asymptotically. Double integrator average consensus differs from first order average consensus in that the nodes' information states converge to the average of their states at the current time rather than the initial time.

Perturbation inputs have been addressed in Section IVE. In Section V, an algorithm was shown where a known input can be used to accurately track the center of a vehicle formation given that each vehicle knows its own position and velocity.

Lastly, there is a need to increase the time between communication intervals. As it stands (3) must have a communication interval that is bounded above as (32). This is very restrictive. Work is needed so that this communication interval can be increased. Perhaps changing the consensus update by decoupling the information state dynamics from the consensus dynamics is necessary.

\section{REFERENCES}

[1] A. Jadbabaie, J. Lin, and A. Morse, "Coordination of groups of mobile autonomous agents using nearest neighbor rules," IEEE Trans. Autom. Control, vol. 48, no. 6, pp. 988-1001, 2003.

[2] R. Olfati-Saber and R. M. Murray, "Consensus Problems in Networks of Agents with Switching Topology and Time-Delays," IEEE Trans. Autom. Control, vol. 49, no. 9, pp. 1520-2533, September 2004.

[3] Z. Lin, M. Broucke, and B. Francis, " Local control strategies for groups of mobile autonomous agents ," IEEE Trans. Autom. Control, vol. 49, no. 4, pp. 622-629, 2004.

[4] W. Ren and R. W. Beard, "Consensus seeking in multiagent systems under dynamically changing interaction topologies," IEEE Trans. Autom. Control, vol. 50, no. 5, pp. 655-661, May 2005.

[5] L. Moreau, "Stability of Multiagent Systems With Time-Dependent Communciation Links," IEEE Trans. Autom. Control, vol. 50, no. 2 , pp. 169-182, 2005.

[6] D. B. Kingston and R. W. Beard, "Discrete-time average-consensus under switching network topologies," in Proceedings of the American Control Conference, 2006.

[7] W. Ren, "On consensus algorithms for double-integrator dynamics," IEEE Trans. Autom. Control, 2008, (to appear).

[8] W. Ren and R. W. Beard, Distributed Consensus in Multi-vehicle Cooperative Control, ser. Communications and Control Engineering. London: Springer-Verlag, 2008.

[9] S. Roy, A. Saberi, and K. Herlugson, "Formation and Alignment of Distributed Sensing Agents with Double-Integrator Dynamics," in Sensor Network Applications. IEEE Press, 2007.

[10] W. Ren and E. Atkins, "Second-order Consensus Protocols in Multiple Vehicle Systems with Local Interactions," Proceedings of the AIAA Guidance, Navigation, and Control Conference, 2005.

[11] J. Fax and R. Murray, "Information flow and cooperative control of vehicle formations," IEEE Trans. Autom. Control, vol. 49, no. 9, pp. 1465-1476, 2004.

[12] Z. Lin, B. Francis, and M. Maggiore, "Necessary and sufficient graphical conditions for formation control of unicycles," IEEE Trans. Autom. Control, vol. 50, no. 1, pp. 121-127, 2005.

[13] G. Lafferriere, A. Williams, J. Caughman, and J. Veerman, "Decentralized control of vehicle formations," Systems and Control Letters, vol. 54, no. 9, pp. 899-910, 2005.

[14] W. Ren, "Consensus strategies for cooperative control of vehicle formations," IET Control Theory \& Applications, vol. 1, no. 2, pp. 505-512, March 2007.

[15] R. Olfati-Saber, "Flocking for multi-agent dynamic systems: algorithms and theory," IEEE Trans. Autom. Control, vol. 51, no. 3, pp. 401-420, 2006.

[16] H. Tanner, A. Jadbabaie, and G. Pappas, "Flocking in Fixed and Switching Networks," IEEE Trans. Autom. Control, vol. 52, no. 5, pp. 863-868, 2007.

[17] W. L. Brogan, Modern Control Theory (3rd ed.). Prentice-Hall, Inc., 1991, ch. 7, pp. 245-281.

[18] A. Morse, "Supervisory control of families of linear set-point controllers Part I. Exact matching," IEEE Trans. Autom. Control, vol. 41, no. 10, pp. 1413-1431, 1996.

[19] W. J. Rugh, Linear system theory (2nd ed.). Upper Saddle River, NJ, USA: Prentice-Hall, Inc., 1996. 